The Fermi gas and liquid-drop models lead to considerably higher energies $(500 \mathrm{MeV}$. or more) for $\bar{T}=5 \mathrm{MeV}$.

It is interesting to note that in the pure Bohr type of process the particles should be emitted under arbitrary angles; however, the angular distribution of tracks in a number of the stars show marked deviations from spherical symmetry, and probably these disintegrations are characterized by the fact that thermal equilibrium is never fully attained. Fig. 3 shows an extreme example of such a star. The probability that all four tracks were ejected under arbitrary angles is calculated as less than 1 in $10^{5}$. However, since the recoil nucleus shows considerable momentum, and the ejected particles are of relatively low energy ( 20 or $30 \mathrm{MeV}$.), it is doubtful if this process is of the pure Heisenberg type, as observed in cloud chambers ${ }^{3}$.

I should like to express my sincere thanks to Dr. J. L. Michiels, for a valuable discussion of the above material.

Imperial College of Science and Technology, London, S.W.7. July 12.

${ }^{1}$ Weisskopf, Phys. Rev., 52, 295 (1937).

${ }^{2}$ Lattes, Muirhead, Occhialini and Powell, Nature, 159, 694 (1947). ${ }^{3}$ Hazen, Phys. Rev., 65, 67 (1944).

\section{Adsorption Isotherms from Chromatographic Measurements}

IT has been shown ${ }^{1}$ that adsorption isotherms can be calculated with good accuracy from chromatographic data, utilizing the measured concentrations $\left(c_{x}\right)$ of eluted solute after developing the original band with $v$ c.c. of pure solvent. Use is made of the equation

$$
q=f_{(c)}=\left(\mu_{c}+v \cdot c_{v}\right) / x_{t}-\alpha \cdot c_{v},
$$

where $q$ is the amount of solute adsorbed per gm. of adsorbent, $x_{t}$ is the total amount of adsorbent in the column (gm.), $\alpha$ is the pore space of the adsorbent (c.c./gm. adsorbent), $\mu_{c}$ is the total eluate after the point of concentration $c$.

In the case of strongly coloured solutes, however, where the movement of the front boundary of the chromatographic band can be followed easily, it is simpler to obtain the adsorption isotherm from the volume $v$ of pure solvent used and from the amount of adsorbent $x_{f}$ through which the coloured front has travelled from the top of the column.

The position of the front is governed by equation 1 in the form:

$$
m=q_{f} \cdot x_{f}-\left(v-\alpha x_{f}\right) \cdot c_{f},
$$

where $m$ is the total amount of solute used. With $m, v$ and $x_{f}$ known, equation 2 represents a linear relationship between $q$ and $c$ with a gradient $d q / d c=$ $\left(v-\alpha . x_{f}\right) / x_{f}$. However, as shown by De Vault ${ }^{2}$ and by Weiss $^{3}$

$$
\left(v-\alpha \cdot x_{f}\right) / x_{f}=\left[d f_{(c)} / d c\right] c=c t .
$$

Thus equation 2 , taken as a function of the variables $q$ and $c$, corresponds to the tangent to the adsorption 1sotherm $q=f_{(c)}$ at the point $q_{f}, c_{f}$, and, with a sequence of coexistent values of $v$ and $x_{f}$, equation 2 gives a family of straight. lines, from which the adsorption isotherm can be easily constructed as the envelope.

The relative accuracy of the equilibrium values so obtained is approximately that of $v$ and $x_{f}$, which, for a well-made column, can be determined to at

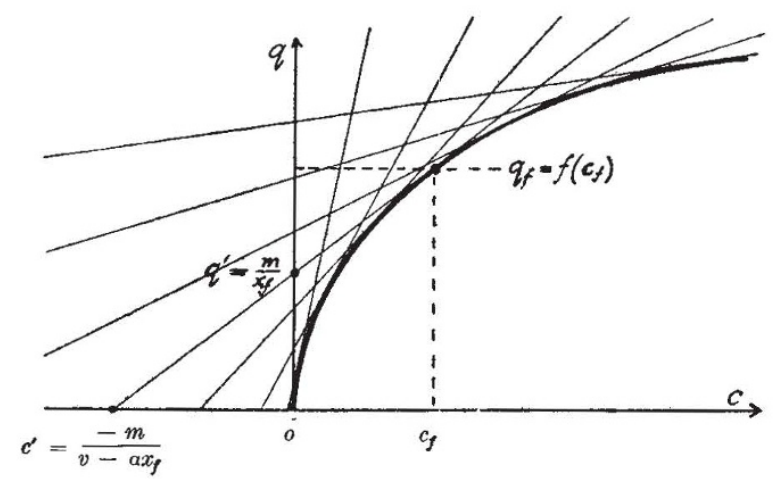

ADSORPTION ISOTEERM AS ENVWLOPE OF THE FUNCTION $m=x_{j} \cdot q-\left(v-a \cdot x_{f}\right) . c$

least \pm 1 per cent. This method thus gives the complete adsorption isotherm without requiring a single concentration measurement by just following the movement of the front boundary, and this with an accuracy at least equal to that of the best classical methods of measuring adsorption equilibria.

Science Laboratory,

E. Glueckauf

University of Durham.

May 24.

${ }^{1}$ Glueckauf, E., Nature, 156, 748 (1945). Experimental details in J. Chem. Soc., in the press.

- De Vault, D., J. Amer. Chem. Soc., 65, 532 (1943).

${ }^{3}$ Weiss, J., J. Chem. Soc., 297 (1943).

\section{Slip Flow in Granular Beds}

The Poiseuille type of equation for capillaries of any shape takes the form,

$$
u_{e}=\frac{m^{2}}{k_{0} \eta} \cdot \frac{\Delta p}{L_{e}} \cdot \cdot \cdot . \quad .
$$

for capillaries of any cross-section, where $k_{0}=2$ and $m=r / 2$ for a circular capillary of radius $r$, and $k_{0}$ has generally values between 2 and 3 for other shapes ${ }^{1}$. Application of this to granular beds follows by putting $u=u_{e} \cdot \varepsilon \cdot L / L_{e}, m=\varepsilon / S \sigma(1-\varepsilon), k=$ $k_{0} \cdot\left(L_{e} / L\right)^{2}$, whence

$$
u=\frac{\varepsilon^{3}}{(1-\varepsilon)^{2}} \cdot \frac{\Delta p}{k \eta S^{2} \sigma^{2} L} \cdot . \quad .
$$

$k$ is known as Kozeny's constant, and is approximately equal to $5^{1,2}, \Delta p$ is pressure drop in dynes $/ \mathrm{cm}^{2}$, and other symbols are as in the paper of Lea and Nurse ${ }^{2}$.

When slip takes place in a capillary, giving a velocity, $u_{0}$, at the wall, this must be added to the right-hand side of (1). Now, by balancing momentum transfer and pressure difference, Millikan ${ }^{3}$ obtained an expression for $u_{0}$ in a circular capillary in terms of a coefficient of external friction, $\beta$. In brief, tangential force on wall, $F^{\prime}=2 \pi r L_{e} \beta u_{0}=\pi r^{2} . \Delta p$. This is readily generalized to non-circular capillaries, giving

$$
u_{0}=\frac{m}{\beta} \cdot \frac{\Delta p}{L_{e}}
$$

The Poiseuille type of equation, corrected for slip, thus becomes

$$
u_{e}=\frac{m^{2}}{k_{0} \eta} \cdot \frac{\Delta p}{L_{e}} \cdot\left(1+\frac{k_{0} \eta}{m \beta}\right) \cdot .
$$

Millikan further showed that $\beta$ is given by

$$
\beta=\frac{1}{2} p \bar{c} \div\left(\frac{2}{f}-1\right) . . \quad \text {. }
$$

